\title{
EDITORIAL
}

\section{IN THE AUGUST 2009 ISSUE OF CLINICS}

\author{
Mauricio Rocha-e-Silva, Editor
}

doi: $10.1590 / \mathrm{S} 1807-59322009000800001$

In this August 2009 issue of CLINICS we highlight a newly proposed solution for the problem of optimizing rheological conditions for coronary bypass grafts, under development by Oliveria et al: A U-shaped aortic flap $2 \mathrm{~cm}$ $\mathrm{x} 4 \mathrm{~cm}$, pedicled to the aorta through its cranial end, had its rims sutured one to the other in order to create a conduit with a length of $4 \mathrm{~cm}$ and an internal diameter of $4 \mathrm{~mm}$. The rest of the aortotomy was corrected by a direct suture between its rims. A radial artery or a saphenous vein graft may be sutured end-to-end to this "in situ" aortic wall graft theoretically providing an ideal rheological flow profile.

In this issue CLINICS publishes one more basic research and 9 clinical science papers.

1. Haddad et al. analyzed the visual response, the use of optical correction and prescribed low vision aids in a population of 100 children with congenital glaucoma and conclude that a careful assessment and the prescription of optical corrections and low vision aids are mandatory in children with congenital glaucoma, since this will assist their global development, improving efficiency in their daily life activities and promoting social and educational inclusion.

2. Scerni et al. note that in Brazil, patients with chronic myeloid leukemia (CML) in the chronic phase were not given first-line imatinib treatment until 2008, and describe the results the treatment of 44 patients with chronic phase CML with second-line imatinib therapy; they conclude that patients in the chronic phase exhibit a higher probability of achieving and maintaining major molecular remission if the interval between diagnosis and initiation of therapy was shorter than one year.

3. Carvalho et al. evaluated self-medication used as initial treatment by ophthalmologic patients through a cross-sectional analytic survey of 561 consecutive patients seen in the ophthalmology emergency room of a teaching hospital and conclude that patients often attempt to treat

Hospital das Clínicas, Faculdade de Medicina da Universidade de São Paulo - São Paulo/SP, Brazil.

mrsilva36@hcnet.usp.br conditions that require ophthalmologic emergency care by self-medicating with homemade or manufactured products. The most widely used products include boric acid, normal saline, leaf infusions and breast milk. This behavior occurs independently of educational level, gender, age or the nature of the ocular condition. They find that self-medication is a culturally driven practice that is used even in cases of acute ocular injuries.

4. Yazbeck et al. compared the 20 month evolution of 60 patients submitted to two surgical techniques (denervation levels T2 or T3) for sympathectomy using video-assisted thoracoscopy to treat palmar hyperhidrosis. Resolution occurred in 59 cases (failure in 1 T3 denervation), but 20 months after the procedure both groups presented some degree of compensatory hyperhidrosis which was less severe in the T3 group. Quality of life did not differ between the two groups.

5. Zurbaran et al. compared two questionnaires (Portuguese version of the World Health Organization Quality of Life Assessment-Bref vs. Multicultural Quality of Life Index) in their ability to assess maternal quality of life of 101 women in the postpartum period in southern Brazil, and found that a significant correlation occurs between the Multicultural Quality of Life Index and the four domains of the WHO quality of life scores They also found that the Psychological and Environment domains of the WHO questionnaire reveals differences related to different socio-economic strata, thus suggesting that it may be more sensitive than the multicultural questionnaire in detecting the impact of socioeconomic status.

6. Kayalar et al. evaluated how bone mineral density in the calcaneus measured by a dual energy X-ray laser correlates with bone mineral density in the spine and hip in 2,884 Turkish women over 40 years of age; they also investigated whether calcaneal dual energy X-ray laser variables are associated with clinical risk factors to the same extent as axial bone mineral density measurements obtained using dual energy x-ray absorbtiometry. They results indicate that smokers with a positive history of fracture, hormone replacement 
therapy (HRT), covered dressing style, lower educational level, no regular exercise habits, and low tea consumption exhibited significantly lower calcaneal dual energy X-ray laser readings.

7. Oliveira et al. endeavored to establish a relationship between vascular calcifications seen via mammography and coronary artery disease, to estimate the risk ratios, and to investigate the interrelationships between vascular calcification and other risk factors for coronary artery disease. They conclude through a case-control study conducted in 40 women with, versus 40 women without a history coronary artery disease that vascular calcifications seen on mammography constitute an independent factor indicating coronary artery disease.

8. Bisaccioni et al. retrospectively analyzed co-morbidities present in the medical records of 245 patients with severe asthma who represent circa $10 \%$ of all asthma patients and found that most common occurrences are rhinitis, gastroesophageal reflux disease, vocal cord dysfunction (VCD), nasal polyposis and bronchiectasis.

9. Ribeiro Junior et al. reviewed medical records and radiology files of 28 patients with a proven diagnosis of hepatocellular adenoma, an exceedingly rare life-threatening condition and report a series of emergency surgeries performed at their institution for this condition. The surgical approaches were as follows: right hemihepatectomy for controlling intraperitoneal bleeding, and right extended hepatectomy and non-anatomic resection of the liver for intrahepatic hemorrhage. There were no deaths, and the postoperative complications were bile leakage and wound infection (reoperation), as well as intraperitoneal abscess (re-operation) and pleural effusion. They conclude that spontaneous ruptured hepatocellular adenoma may be treated by surgery for controlling hemorrhages and stabilizing the patient, and the decision to operate depends upon both the patient's condition and the expertise of the surgical team.

10. Pompeu et al. performed a prospective study on 16 elderly males to evaluate the effects of androgen deprivation therapy on body composition and resting metabolic rate in patients with prostate cancer; they report that body weight, lean mass, and lipid oxidation decreased significantly, whereas carbohydrate oxidation, FSH and LH levels increased significantly. Changes in fat mass and bone mineral density were not significant.

11. Moraes-Filho et al. studied the prevalence of comorbidities in 670 patients suffering from gastroesophageal reflux and found a total of 1,664 instances of comorbidities recorded in 586 patients ( $87.5 \%$ of patients); the most common were arterial hypertension (21\%), hypercholesterolemia (9\%), obesity (9\%), type II diabetes mellitus (5\%) and depression (4\%). Two or more comorbidities were present in 437 individuals $(64.8 \%)$. The occurrence of comorbidities increased with age and was higher in patients with the nonerosive form of the disease.

12. Moya et al. endeavored to determine whether a quiet static standing posture is a predictor of compensatory postural adjustment in 20 healthy individuals standing on a movable surface who underwent unexpected, standardized backward and forward postural perturbations while kinematic data were acquired. No relationship between the position of the body segments in the initial and final frames and the associated displacement of the body segments was identified.

13. Coelho da Mota et al. investigated the skin flap viability through orthogonal polarization spectral imaging and effects of buflomedil and pentoxifylline, both of which are used in reconstructive surgery of hamster skin flap microcirculation and found that functional capillary density values were higher in the buflomedil group compared to the control and pentoxifylline groups in this model. Functional capillary density was increased by buflomedil and pentoxifylline, but did not influence distance from the skin flap base to blood flow cessation or the percentage of viable skin. The technique using buflomedil showed favorable potential to assess/ predict the viability of skin flaps within $1 \mathrm{~h}$ after surgery.

Clinics further publishes a review on the history of blood substitutes and 3 case reports. 